2016

\title{
Is delirium being detected in emergency?
}

Victoria Traynor

University of Wollongong, vtraynor@uow.edu.au

Nicholas Cordato

University of New South Wales

Pippa Burns

University of Wollongong, pippa@uow.edu.au

Yun Xu

University of New South Wales

Nicole Britten

University of Wollongong, nbritten@uow.edu.au

See next page for additional authors

\section{Publication Details}

Traynor, V., Cordato, N., Burns, P., Xu, Y., Britten, N., Duncan, K., deVries, L. \& Mckinnon, C. (2016). Is delirium being detected in emergency?. Australasian Journal on Ageing, 35 (1), 54-57.

Research Online is the open access institutional repository for the University of Wollongong. For further information contact the UOW Library: research-pubs@uow.edu.au 


\title{
Is delirium being detected in emergency?
}

\begin{abstract}
Objective To report on the use of Delirium Care Pathways to screen for and recognise delirium by Aged Care Services in Emergency Teams (ASETs) at five metropolitan hospitals in New South Wales, Australia.

Knowledge of delirium and the use of Delirium Care Pathways are vital to ensure that older people presenting with delirium receive best practice care. Methods An audit of 205 randomly selected medical records of clients over 65 years presenting to an ASET was conducted. Results Delirium was recorded in the medical records notes of four clients (2\%). However, the auditors identified another 27 clients with symptoms of delirium. Conclusions Delirium is still frequently undiagnosed and misdiagnosed in older people presenting to emergency departments. This indicates a need for further education and professional development for and by health-care practitioners. Only with greater awareness of delirium will the care and health outcomes of older adults presenting with delirium in acute care settings improve.
\end{abstract}

\section{Disciplines}

Medicine and Health Sciences | Social and Behavioral Sciences

\section{Publication Details}

Traynor, V., Cordato, N., Burns, P., Xu, Y., Britten, N., Duncan, K., deVries, L. \& Mckinnon, C. (2016). Is delirium being detected in emergency?. Australasian Journal on Ageing, 35 (1), 54-57.

\section{Authors}

Victoria Traynor, Nicholas Cordato, Pippa Burns, Yun Xu, Nicole Britten, Kim Duncan, Loren deVries, and Colleen Mckinnon 


\section{Is Delirium Being Detected in Emergency?}

Victoria Traynor

School of Nursing, University of Wollongong, Wollongong, New

South Wales, Australia

Nicholas Cordato

St George and Calvary Hospitals; and The University of New South

Wales, Sydney, New South Wales, Australia

Pippa Burns

School of Nursing, University of Wollongong, Wollongong, New South Wales, Australia

Yun Xu

St George and Calvary Hospitals; and The University of New South

Wales, Sydney, New South Wales, Australia

Nicole Britten

School of Nursing, University of Wollongong, Wollongong, New

South Wales, Australia

Kim Duncan

St Vincent's Health Network, Sydney, New South Wales, Australia

Loren DeVries

Garrawarra Centre, Helensburgh, South Eastern Sydney, Local

Health District, New South Wales, Australia

Colleen McKinnon

South East Sydney Local Health District, Sydney, New South

Wales, Australia 


\section{Abstract:}

Objective(s): To report on the use of Delirium Care Pathways, to screen for and recognise delirium, by Aged Care Services in Emergency Teams (ASET) at five metropolitan hospitals, in New South Wales, Australia. Knowledge of delirium and the use of Delirium Care Pathways are vital to ensure that older people, presenting with delirium receive best practice care.

Methods: An audit of 205 randomly selected medical records of patients over 65 presenting to an ASET was conducted.

Results: Delirium was recorded in the medical records notes of four patients (2\%). However, the auditors identified another 27 patients with symptoms of delirium.

Conclusion(s): Delirium is still frequently undiagnosed and misdiagnosed in older people presenting to Emergency Departments. This indicates a need for further training of health care professionals. Only with greater awareness of delirium will the care and health outcomes of older adults presenting with delirium, in acute care settings, improve.

\section{Key Points}

- Delirium is frequently undiagnosed in older people presenting to Emergency Departments.

- The terms 'confused', 'drowsy' and 'disorientated' are frequently used in medical records to describe patients with a delirium.

- Increased familiarity with current best practice in the recognition, assessment, identification and diagnosis of delirium would ensure appropriate management of older people with a delirium and can prevent the onset of a delirium. 


\section{Introduction}

Delirium is a potentially reversible disorder characterised by the rapid onset of impaired attention, altered cognition, altered consciousness, or changes in attention. These symptoms typically develop quickly over a few hours or days. Delirium results from the interrelationship between risk factors, such as: old age, comorbidity, and polypharmacy, with precipitating factors such as electrolyte disturbance, uncontrolled pain, and sleep deprivation ${ }^{1}$. However, delirium can present in different ways making it difficult to recognise ${ }^{2,3}$. Delirium is associated with increased cognitive and functional decline, longer hospital stays, higher rates of institutionalisation and higher mortality rates ${ }^{1,4-6}$. As such, prompt recognition and identification of delirium upon admission to the Emergency Department (ED) has the potential to improve patients' outcomes and provide significant cost savings to the hospital. In addition, delirium in hospitalized persons is associated with increased rates of falls and incontinence ${ }^{7,8}$

If admitted to hospital, adults aged over 65 years are at significant risk of delirium;, with $15-50 \%$ developing delirium ${ }^{5,6}$. As the signs and symptoms of delirium are non-specific and older adults regularly have concurrent health problems, often of a cognitive nature, delirium can go unrecognized and untreated in hospitals unless staff are trained in its prevention, identification and management ${ }^{9}$. On admission to hospital, all older people should be assessed for additional delirium risk factors and vulnerabilities ${ }^{1}$. As the population ages, it is expected that the incidence of delirium will increase, along with the associated poor health outcomes, if best practice delirium care strategies are not implemented.

In Australia, clinical practice guidelines for the detection, prevention and management of delirium in older people were developed in $2006{ }^{10}$. They indicated that health professionals such as Aged Care Services in Emergency Teams (ASETs) in Emergency Departments should routinely assess the cognitive and behavioural status of older people with the same priority afforded to the measurement of other vital signs. Such assessment would facilitate the early recognition of delirium, reducing adverse outcomes, such as falls and increased mortality, morbidity and hospital length of stay (LOS). In 2011, these guidelines were complemented by the Delirium Care Pathways (DCP) ${ }^{11}$. The DCP recommend the use of the Confusion Assessment Method (CAM) as a tool to distinguish between delirium, dementia and depression ${ }^{12}$. The CAM is completed after a patient interview to provide a baseline measure of cognition. Initial completion of the CAM relies on information obtained from attending family members or carers in order to establish the speed of onset. The CAM should be completed at regular intervals in a similar way to blood pressure readings. The readings can be compared to the baseline reading to indicate fluctuations in cognition. Staff require specific training in the use of CAM ${ }^{4}$. 
During the implementation of the DCP in ASET, it became apparent that many health care practitioners did not fully understand the term 'delirium'. The medical record audit reported here was designed to identify any gaps in the processes used by ASET to recognise and identify delirium. This knowledge is vital to improve the care and healthcare outcomes of older people presenting with delirium in acute care settings, and achieve best practice care.

\section{Methods}

The UK National Institute of Clinical Excellence (NICE) delirium audit tool was used to analyse medical records of older people referred to ASET during an Emergency Department admission ${ }^{13}$. Data collected consisted of: admitting location of the patient, clinical discipline of ASET members, interventions implemented by ASET, recording of cognitive and behavioural changes by ASET, record of a pre-existing cognitive impairment or confirmed diagnosis of dementia; and recording delirium symptoms which were included as a tick box list with space to add symptom descriptors.

The audit was conducted in February/March 2011, on 205 randomly selected medical records of patients over 65 referred to an ASET, at one of five metropolitan hospitals, during September, October and November 2010. Medical records were selected based on a random number generator (random.org). Inter-rater reliability was established by consensus in data collected during the pilot and after the audit. The auditors were senior clinicians from the project team (NC, YX, NB, KD, LDV, $\mathrm{CM}, \mathrm{CB} \& \mathrm{GP}$ ) who were bound to confidentiality by their registration standards. Ethical approval was obtained from the University and Local Health District. All extracted data were de-identified

\section{Results}

The majority of patients whose medical record were audited had been admitted from the community $(78 \%)$, followed by residential accommodation services (14\%). Most patients were assessed by a Registered Nurse (78\%) or Medical Doctor (7\%). The results of the audit indicated that $15 \%$ of patients presented with symptoms of delirium $(n=31)$; but delirium was only recorded in the medical records of four patients (2\%). The auditors, reviewing the medical records, identified an additional 27 patients (13\%) who had symptoms of delirium recorded without delirium being documented in the medical records. Of the four patients where a delirium was recorded, two of the patients had their delirium documented by a Medical Doctor, and one each by a Registered Nurse and Occupational Therapist. 
The terms 'confused', 'drowsy' and 'disorientated' were most frequently used to describe patients with a delirium in their medical records (Table 1).

\section{Chart1: Terminology used to describe delirium in the medical records}

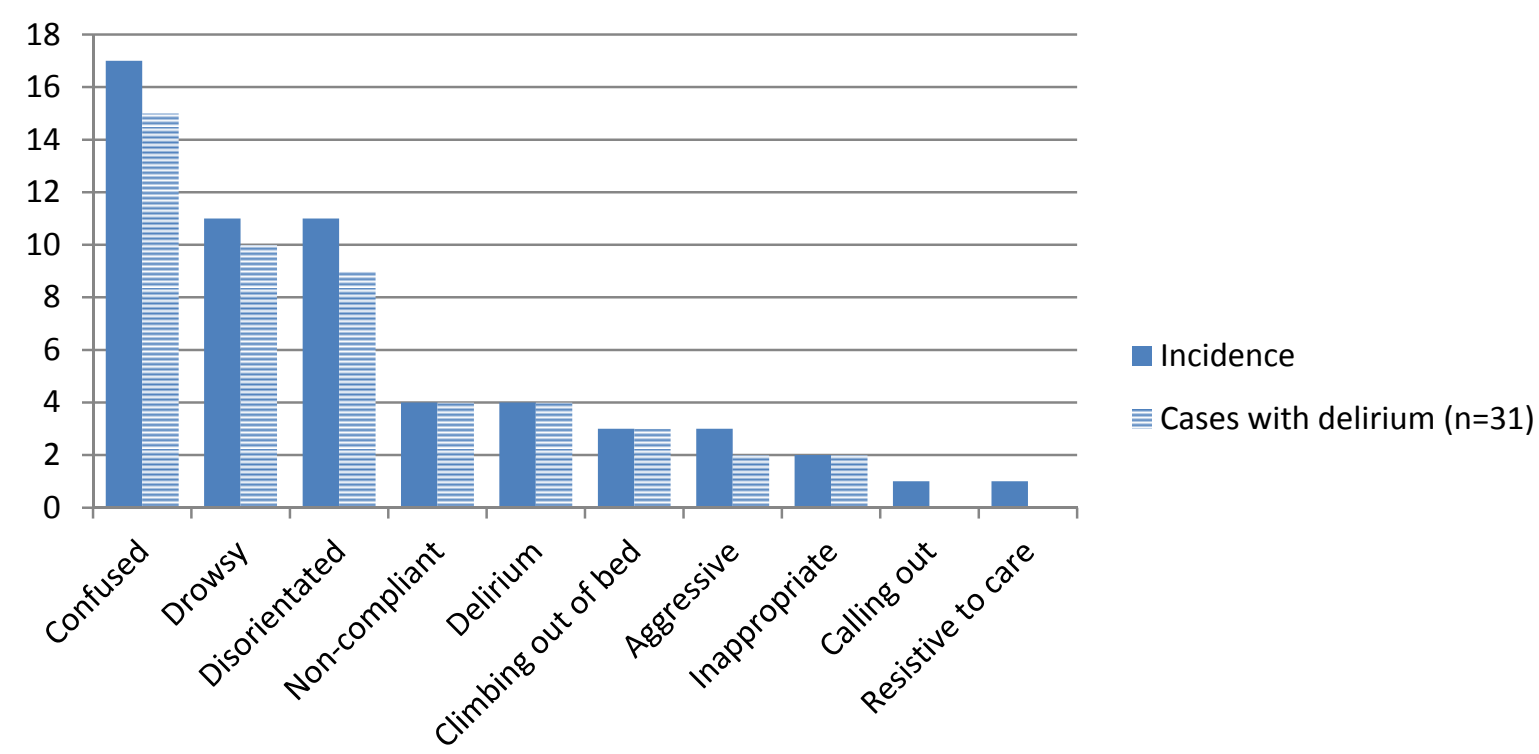

\section{Baseline Assessment Tools}

The cognitive status of patients was noted in just under one-third of cases and the use of cognitive screens was similar (Table 2). Of the 31 patients identified as having delirium by the audit, only three had a CAM score recorded in their medical records; only one of which had a delirium recorded in their medical records (Table 2).

Table 1: A Summary of Assessment Tools and Methods Documented in the Medical Records

\begin{tabular}{|c|c|c|}
\hline & Yes & $\begin{array}{l}\text { Cases } \\
\text { with } \\
\text { delirium } \\
(\mathrm{n}=31)\end{array}$ \\
\hline Usual cognitive status noted & $56(27 \%)$ & $12(39 \%)$ \\
\hline Cognitive Screen conducted & $53(26 \%)$ & $10(32 \%)$ \\
\hline $\begin{array}{l}\text { Confusion Assessment Method (CAM) score } \\
\text { recorded }\end{array}$ & $36(18 \%)$ & $3(10 \%)$ \\
\hline $\begin{array}{l}\text { Cognitive symptoms followed with Delirium } \\
\text { Screen }\end{array}$ & $36(18 \%)$ & $5(16 \%)$ \\
\hline $\begin{array}{l}\text { Cognitive impairment or behavioural } \\
\text { changes noted in file }\end{array}$ & $11(5 \%)$ & $8(26 \%)$ \\
\hline
\end{tabular}




\section{Discussion}

In only $2 \%$ of the medical records reviewed was a delirium documented despite this retrospective audit indicating that the rate of delirium among the patients was much higher, with $14 \%$ of patients recognised as presenting with a delirium $(n=31)$. This incidence rate of delirium (14\%) reflected international hospital data ${ }^{14}$. It is of concern that delirium was not formally documented in the medical records of the majority of the patients who presented with a delirium ( 27 of the 31 patients $-87 \%)$.

Delirium was most frequently documented by a medical practitioner, despite the fact that a Registered Nurse was most likely to have completed the client assessment. This suggests that further investigation into why Registered Nurses and allied health practitioners are not currently documenting delirium is required, including whether there is a need for specific training around delirium. Delirium was frequently identified using descriptive words such as 'confused', 'drowsy and 'disorientated'. It is not clear from these results why these words were used. It is possible that the documenting health care practitioners were not familiar with the presentation of delirium. However, it could be because Registered Nurses are not comfortable recording delirium, as it may be perceived that they are giving a diagnosis. Further investigation is required to identify which factors are contributing to unchanged practice.

Assessment and knowledge of the usual level of cognitive functioning of patients is vital for the early recognition and investigation of delirium, and is crucial to their optimal management and healthcare outcomes. The Delirium Care Pathways recommends that health professionals routinely assess the cognitive and behavioural status of older people, with the same priority afforded to the measurement of other vital signs. However the results show that this does not always occur and that the assessment and management of patients presenting in this study with delirium were not always in accordance with the Delirium Clinical Pathways. Only one of the hospital ASETs audited, used the CAM to screen for delirium among older patients; despite its recommended use in the DCP ${ }^{11}$. The CAM guides diagnosis of delirium and provides an objective measure of current cognitive function ${ }^{2}$. It is a well validated tool ${ }^{15}$ and its lack of use suggests a need for staff education. It should be noted that the Delirium Care Pathways has, since the audit, been superseded by 'A Better Way to Care: Safe and High-Quality Care for Patients with Cognitive Impairment (Dementia and Delirium) in Hospital' ${ }^{16}$. 


\section{Practice Implications}

Older adults presenting to ED are dependent upon ASET teams being familiar with current best practice in the recognition, assessment, identification and diagnosis of delirium.. This not only ensures appropriate management of older people with a delirium but more importantly can prevent the onset of a delirium.

\section{Conclusions}

These audit results indicate that delirium in older people is often undiagnosed or misdiagnosed in the Emergency Departments. There is clearly a need for more training of Emergency Department staff around delirium, and particularly the Delirium Care Pathways. Only with greater awareness will the care and healthcare outcomes of older people presenting with delirium in acute care settings improve.

\section{ACKNOWLEDGEMENTS}

We would like to acknowledge the contribution of both Glenn Power and Christine Banks to this project. 


\section{References}

1. Australian and New Zealand Society for Geriatric Medicine. Position Statement 13: Delirium in Older People.; 2012.

2. Commonwealth of Australia. Delirium in Older People. Canberra, Australia; 2006. Available at: http://www.health.gov.au/internet/main/publishing.nsf/Content/4F8338AEFA71A16ECA257 BF0001F4054/\$File/DeliriuminOlderPeople.pdf.

3. O'Keefe ST, Lavan JN. Clinical significance of delirium subtypes in older people. Age Ageing. 1999;28(1):115-119.

4. Milisen K, Lemiengre J, Braes T, Foreman MD. Multicomponent intervention strategies for managing delirium in hospitalized older people: systematic review. J Adv Nurs. 2005;52(1):7990. doi:10.1111/j.1365-2648.2005.03557.x.

5. Rapp C. Acute confusion / delirium protocol. J Gerontol Nurs. 2001;27(4):21-33.

6. Gleason O. Delirium. Am Fam Physician. 2003;67(5):1027-1035.

7. Lakatos BE, Capasso V, Mitchell MT, et al. Falls in the General Hospital : Association With Delirium , Advanced Age , and Specific Surgical Pro ... Psychosomatics. 2009;50(3):218-226.

8. Inouye SK, Westendorp RGJ, Saczynski JS. Delirium in elderly people. Lancet. 2014;383(9920):911-22. doi:10.1016/S0140-6736(13)60688-1.

9. Burns A, Gallagley A, Byrne J. Delirium. J Neurol Neurosurg Psychiatry. 2004;75(3):362-367. doi:10.1136/jnnp.2003.023366.

10. Australian Health Ministers' Advisory Council: Health Care of Older Australians Standing Committee. Clinical Practice Guidelines for the Management of Delirium in Older People. Melbourne, Victoria; 2006. Available at: www.health.vic.gov.au/acute-agedcare.

11. Australian Health Ministers' Advisory Council: Health Care of Older Australians Standing Committee. Delirium Care Pathways. Canberra, ACT; 2011.

12. Clinical Epidemiology and Health Service Evaluation Unit. Clinical Practice Guideline for the Management of Delirium in Older People. Melbourne; 2006.

13. National Institute for Health and Clinical Excellence. Delirium: Diagnosis, Prevention and Management. Clinical Guidline 103. London; 2010.

14. Barron EA, Holmes J. Delirium within the emergency care setting, occurrence and detection: a systematic review. Emerg Med J. 2013;30(4):263-8. doi:10.1136/emermed-2011-200586. 
15. Wei LA, Fearing MA, Sternberg EJ, Inouye SK. The Confusion Assessment MEthod (CAM): A Systematic Review of Current Usage. J Am Geriatr Soc. 2009;56(5):823-830. doi:10.1111/j.1532-5415.2008.01674.x.The.

16. Australian Commission on Safety and Quality in Healthcare. A better way to care: Safe and high-quality care for patients with cognitive impairment (dementia and delirium) in hospital. 2014. Available at: http://www.safetyandquality.gov.au/our-work/cognitiveimpairment/better-way-to-care/. Accessed April 7, 2015. 